\title{
Silver Nanoparticles Produced by Magnetron Sputtering and Selective Nanodecoration onto $\alpha$-Cyclodextrin/Carboxylic Acid Inclusion Compounds Crystals
}

\author{
Bárbara Herrera $^{1 *}$, Tamara Bruna ${ }^{1}$, Daniel Guerra ${ }^{1}$, Nicolás Yutronic ${ }^{1}$, \\ Marcelo J. Kogan ${ }^{2}$, Paul Jara ${ }^{1^{*}}$ \\ ${ }^{1}$ Department of Chemistry, Faculty of Sciences, University of Chile, Santiago, Chile \\ ${ }^{2}$ Department of Pharmacology and Toxicology, Faculty of Chemical and Pharmaceutical Sciences, \\ University of Chile, Santiago, Chile \\ Email: "pjara@uchile.cl
}

Received December 31, 2012; revised February 1, 2013; accepted February 8, 2013

Copyright (C) 2013 Bárbara Herrera et al. This is an open access article distributed under the Creative Commons Attribution License, which permits unrestricted use, distribution, and reproduction in any medium, provided the original work is properly cited.

\begin{abstract}
In this work, we report the preparation of silver nanoparticles (AgNPs) and the nanodecoration of $\alpha$-cyclodextrin inclusion compounds ( $\alpha$-CD IC) microcrystals that contain palmitic (PAc) and stearic acids (SAc) like guest molecules. These IC provide a suitable environment for nucleation, epitaxial growth and immobilization of AgNPs that were obtained by the magnetron sputtering technique. The use of $\alpha$-CD IC substrates with a specific surface morphology in which the functional group of the guest molecule faces outward preferentially from a crystal plane, is an efficient method for the preparation of AgNPs with a low size dispersion, which is probably due to the high affinity between the functional group of the surfactant carboxylic acid guest with the metal nanoparticles.
\end{abstract}

Keywords: Silver Nanoparticles; Nanodecoration; Cyclodextrin; Inclusion Compounds; Magnetron Sputtering

\section{Introduction}

Decoration of organic crystals with metal nanoparticles is a new area of research and is attracting interest because the specific surface functionalization could have potential applications, for example in the manufacture of devices, due to the interesting combination of properties of metal nanoparticles and organic crystals having crystal planes with a modified surface [1]. Specifically, the AgNPs have important physical and chemical properties, among which are highlighted their interesting optical properties that make them useful in techniques such as: Surface Enhanced Raman Spectroscopy (SERS) [2], in the manufacture of solar cells [3], biosensors [4] and even antibacterial properties which makes them excellent candidates to develop biomedical technologies [5]. These nanoparticles can adhere selectively to a specific crystal plane of $\alpha$-CD IC [6,7], which are excellent candidates to become part of these organic-inorganic materials due to their unique properties of interface.

${ }^{*}$ Corresponding authors.
CDs crystallize in two main categories of crystal packing-channel and cage structures which are dependent on the type of cyclodextrin and guest molecules that are used [8-12]. We have reported that in the presence of long-chain alkylamines or alkylthiol guest molecules, $\alpha$ - and $\gamma$-CDs will crystallize exclusively in the channel-like structure, where the guest molecule are located inside the cavity $[13,14]$. Additionally, in a previous paper, we reported the structural determination of $\alpha$-CD IC with decanoic acid as guest molecule using $\mathrm{X}$-ray single crystal diffraction, where the aliphatic chain of the carboxylic acid was threaded through a head-to-head dimer of two cyclodextrin residues in a 1:2 stoichiometry [15]. Recently, we discovered that the inclusion of alkyl-guests can cause the cyclodextrin IC to crystallize as well-defined single crystals. These supramolecular assemblies constructed by non-covalent bonds range in size between nanometers to micrometers, but the shapes of these crystals are essentially identical and independent of the size [13]. Moreover, the IC crystals are anisotropic polyhedra, and the crystal faces provide interfaces with different chemical properties due to the symmetric 
shape of the cyclodextrins and the anisotropic arrangement of the organic guest molecules in the single crystals. The organic interfaces of the anisotropic single crystals are useful for supramolecular chemistry, which has attracted growing interest in the anisotropic decoration with nanoparticles [7].

On the other hand, we reported the preferred deposition of gold nanoparticles (AgNPs) onto microcrystal faces of $\alpha-\mathrm{CD} /$ dodecanethiol and $\alpha-\mathrm{CD} /$ octanethiol ICs $[16,17]$. The immobilization of AgNPs obtained by magnetron sputtering resulted from favorable interaction with the free $-\mathrm{SH}$ groups of the guest molecules. The preferential deposition on the $\{001\}$ plane of the $\alpha$-CD IC crystal occurred because the $-\mathrm{SH}$ groups of the guest molecules within the $\alpha$-CD IC protruded from that plane only. Additionally, recently we reported the synthesis of $\alpha-C D$ ICs with carboxylic acids (octanoic, decanoic, dodecanoic, tetradecanoic, hexadecanoic and octadecanoic). $\alpha$ CD complexes showed a channel-type structure and the stoichiometric ratio between the $\alpha-\mathrm{CD}$ and carboxylic acid were a function of the chain length of the guest molecules. The nanodecoration of these compounds was realized with a dispersion of silver nanoparticles [18]. AgNPs were prepared by addition of sodium borohydride $\left(\mathrm{NaBH}_{4}\right)$ as a reducing agent and thiosalicylic acid (TSA) as a stabilizer into the $\mathrm{Ag}^{+}$solution according to an established protocol [19].

In this paper, we report the selective nanodecoration of $\alpha$-CD ICs (i.e., $\alpha$-CD with hexadecanoic or palmitic acid ( $\alpha-\mathrm{CD} / \mathrm{PAc}$ ) and $\alpha-\mathrm{CD}$ with octadecanoic or stearic acid $(\alpha-\mathrm{CD} / \mathrm{SAc}))$ along the $\{001\}$ crystal plane with AgNPs using the magnetron sputtering technique.

\section{Methods and Materials}

\subsection{Preparation of $\alpha$-Cyclodextrin/Carboxylic Acids Inclusion Compounds}

All reagents were purchased from Sigma-Aldrich with purity between $90 \%-99.9 \%$. The $\alpha$-CD IC with PAc and Sac were obtained directly by mixing carboxylic acid with a saturated solution of $\alpha$-CD in water at room temperature in a 1:1 molar ratio. The immediate precipitation of a white solid indicated the formation of the IC. After $72 \mathrm{~h}$, the IC microcrystals were filtered and dried under a vacuum.

\subsection{Characterization of $\alpha$-Cyclodextrin/ Carboxylics Acids Inclusion Compounds}

Previously, $\alpha-\mathrm{CD} /$ carboxylic acids IC microcrystals were characterized by Proton Nuclear Magnetic Resonance (1H-NMR) in FT/NMR Bruker $400 \mathrm{MHz}$ equipment and by Powder X-ray Diffraction (Powder XRD) in a Siemens D-5000 Diffractometer. Full details of the measurements are in the Supplementary material.

\subsection{Synthesis of Silver Nanoparticles}

To prepare AgNPs, $\alpha-\mathrm{CD} /$ carboxylic acids IC microcrystals were spread on a glass surface to form a homogeneous layer prior to exposure to the sputtering equipment (Magnetron Sputter Coater PELCO SC-6). AgNPs (Ag target, $99.9 \%$ purity) were deposited onto the substrate under an inert atmosphere at room temperature with 25 $\mathrm{mA}$ of current, 0.06 mbar of vacuum and $20 \mathrm{~s}$ of exposure time.

\subsection{UV-Vis Analysis}

The UV-Vis spectra of the AgNPs onto $\alpha-\mathrm{CD} / \mathrm{PAc}$ and $\alpha-\mathrm{CD} / \mathrm{SAc}$ in the solid state were measured with $\mathrm{BaSO}_{4}$ as standard on a Shimadzu UV-2450 Spectrophotometer from $400-800 \mathrm{~nm}$ (1 nm intervals).

\subsection{Transmission Electron Microscopy (TEM), Scanning Electron Microscopy (SEM) and Analysis Energy Dispersive X-Ray Spectroscopy (EDAX)}

AgNPs were observed by TEM using a JEOL 1010 Electron Microscope at $80 \mathrm{kV}$. The samples were prepared by dropping of dispersion of $\alpha$-CD/carboxylic acid IC with AgNPs in 30\% ethanol solution onto Formvar carboncoated copper micro grids and letting them dry.

SEM images of selective nanodecoration of $\alpha-\mathrm{CD} /$ carboxylic acid IC with AgNPs, were acquired with a SEM FE S-4100 Hitachi which was fitted with a field emission source and operated at $20 \mathrm{kV}$. To prepare the samples, nanodecorated IC microcrystals were spread on the sup- port-samples.

The presence of AgNPs onto specific crystal plane of $\alpha-\mathrm{CD} /$ carboxylic acid IC was confirmed by EDAX in a SEM FEI Quanta 200 equipment with GENESIS EDAX detector (See Supplementary material).

\section{Results and Discussion}

\subsection{UV-Visible Spectra}

The optical properties of AgNPs were characterized by UV-visible in solid state. Figure 1 shows the diffuse reflection spectroscopy analysis of AgNPs formed by sputtering deposition onto the specific $\{001\}$ crystal plane of $\alpha-\mathrm{CD} /$ carboxylic acid IC. The characteristic surface plasmon resonance can be observed with absorption maxima at $470 \mathrm{~nm}$ and $439 \mathrm{~nm}$ for the AgNPs onto $\alpha-\mathrm{CD} / \mathrm{PAc}$ and $\alpha-\mathrm{CD} / \mathrm{SAc}$, respectively. Compared to the absorption peaks of dispersion of AgNPs $(475 \mathrm{~nm})$ stabilized by TSA [19]. The plasmon bands observed for AgNPs formed by sputtering deposition were shifted to lower wavelengths and were narrower.

The hypsochromic shift of the plasmon resonance was assigned to a smaller particle size, a smaller dipolar cou- 
pling between particles in close proximity and a change in the dielectric environment. Furthermore, AgNPs aggregates were assumed to be absent, as evidenced by the narrowing of the absorption band and SEM images.

\subsection{SEM and EDAX Analysis}

SEM images indicated that AgNPs were deposited preferentially onto selective crystal face. A set of SEM images that approached the microcrystal are shown in Figure 2. Self-assembly of the AgNPs were observed on the surface of the IC. The sizes of the AgNPs that were deposited were estimated from the exposure time of the substrate $(20 \mathrm{~s})$. These results indicated that the particles did not form in the plasma phase prior to deposition, but formed on the $\alpha-\mathrm{CD} /$ carboxylic acids IC.

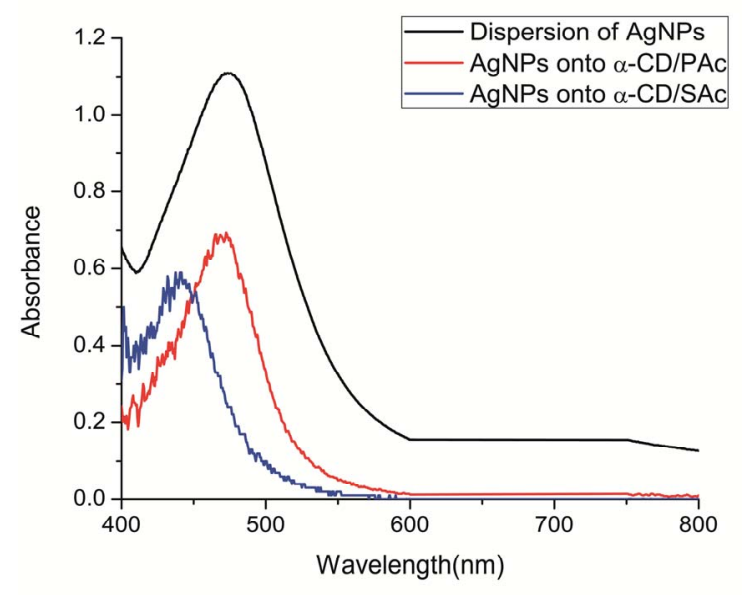

Figure 1. UV-Vis spectra AgNPs obtained onto $\alpha$-CD/PAc and $\alpha-\mathrm{CD} / \mathrm{SAc} \mathrm{IC}$ crystals (red and blue lines, respectively) compared with UV-visible spectra of dispersion of AgNP (black line).

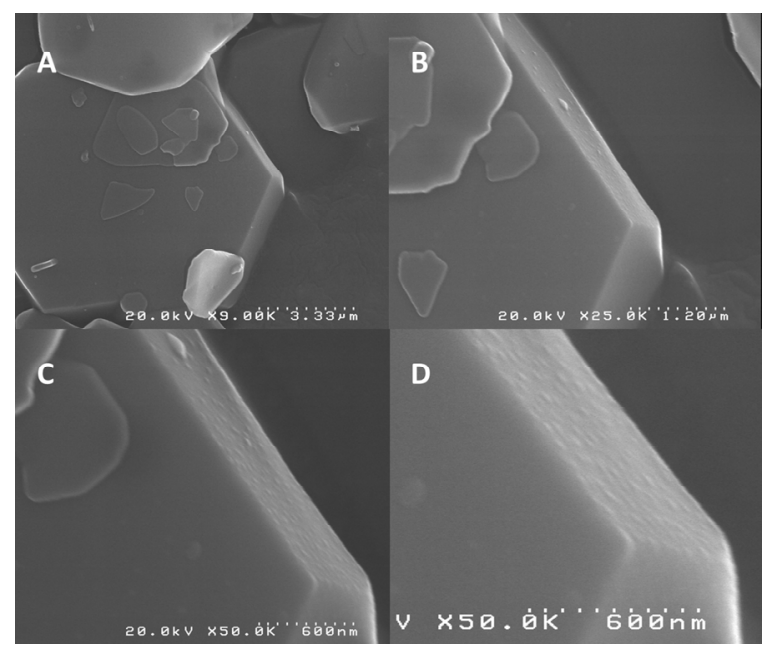

Figure 2. (A), (B), (C) and (D) High resolution SEM images shows the crystal IC shape and preferential deposition of AgNPs onto $\{001\}$ crystal plane.
The $\alpha-\mathrm{CD} /$ carboxylic acid complex structure had a hexagonal structure, and the carboxylic acid molecules were ordered along the $\mathrm{c}$ axis, which resulted in a specific surface that was assumed to be the $\{001\}$ Miller plane. The anchoring of AgNPs to the surface of the crystal was due to the interaction of $\mathrm{Ag}$ with the HOOCgroups of the guest molecule of the IC, which was located at the entrance to the cavity of the $\alpha-\mathrm{CD}$ that corresponded to the $\{001\}$ crystal plane (See Figure 3). This led to the stabilization of the AgNPs on the surface. Therefore, the specific crystal plane provided a convenient method to synthesize and store AgNPs without aggregation.

Figure 3 shows the SEM images of hexagonal micrometer-sized single crystals, which have well-defined shapes with sharp edges. In addition, to investigate the face-selective adhesion of nanoparticles on the $\{001\}$ crystal planes, experiments regarding the decoration of the micrometer-sized crystals were performed by SEM analysis. The EDAX spectra inserted in Figure 3 shows the presence of $\mathrm{Ag}$ from AgNPs onto the crystal surface and indicate the presence of $\mathrm{C}$ and $\mathrm{O}$ from the organic composition of the $\alpha-\mathrm{CD} /$ carboxylic acid IC crystals and $-\mathrm{COOH}$ group that interacts with the AgNPs. The presence of $\mathrm{S}$ is from the carbon ribbon (See Supplementary material). Interestingly, the deposition of nanoparticles was observed only on one preferred face. These results indicate that metal NPs selectively interacted with the $\{001\}$ faces.

The affinity between the silver and $-\mathrm{COOH}$ groups was determined to be greater than the affinity between

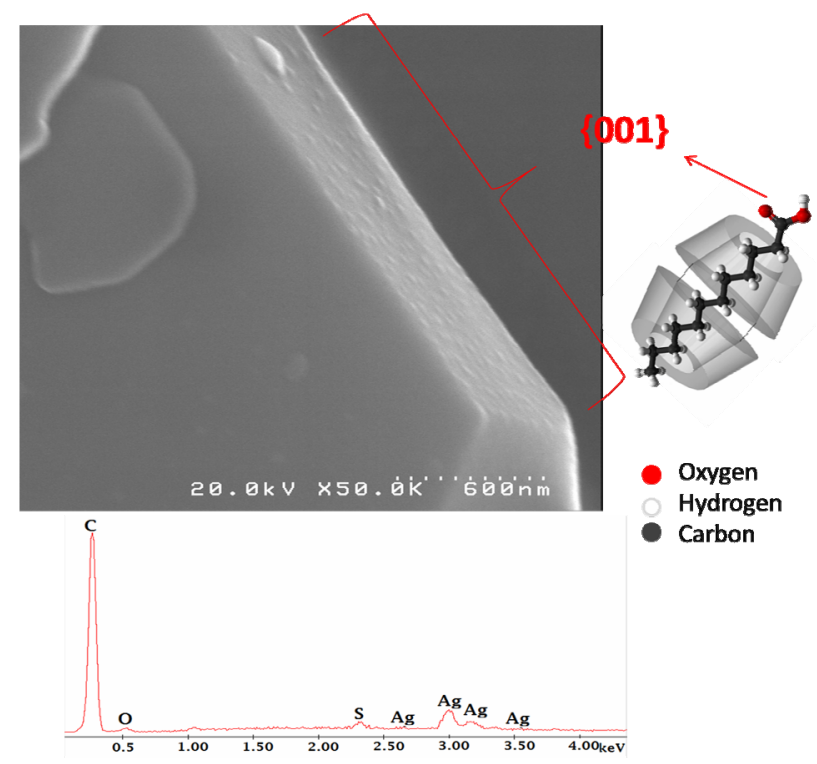

Figure 3. Schematic representation of $2 \alpha \mathrm{CD}$ - carboxylic acid complex basic structure insert onto SEM image that show the selective deposition of particles and EDAX analysis that confirm the presence of $\mathrm{Ag}$ from AgNPs. 

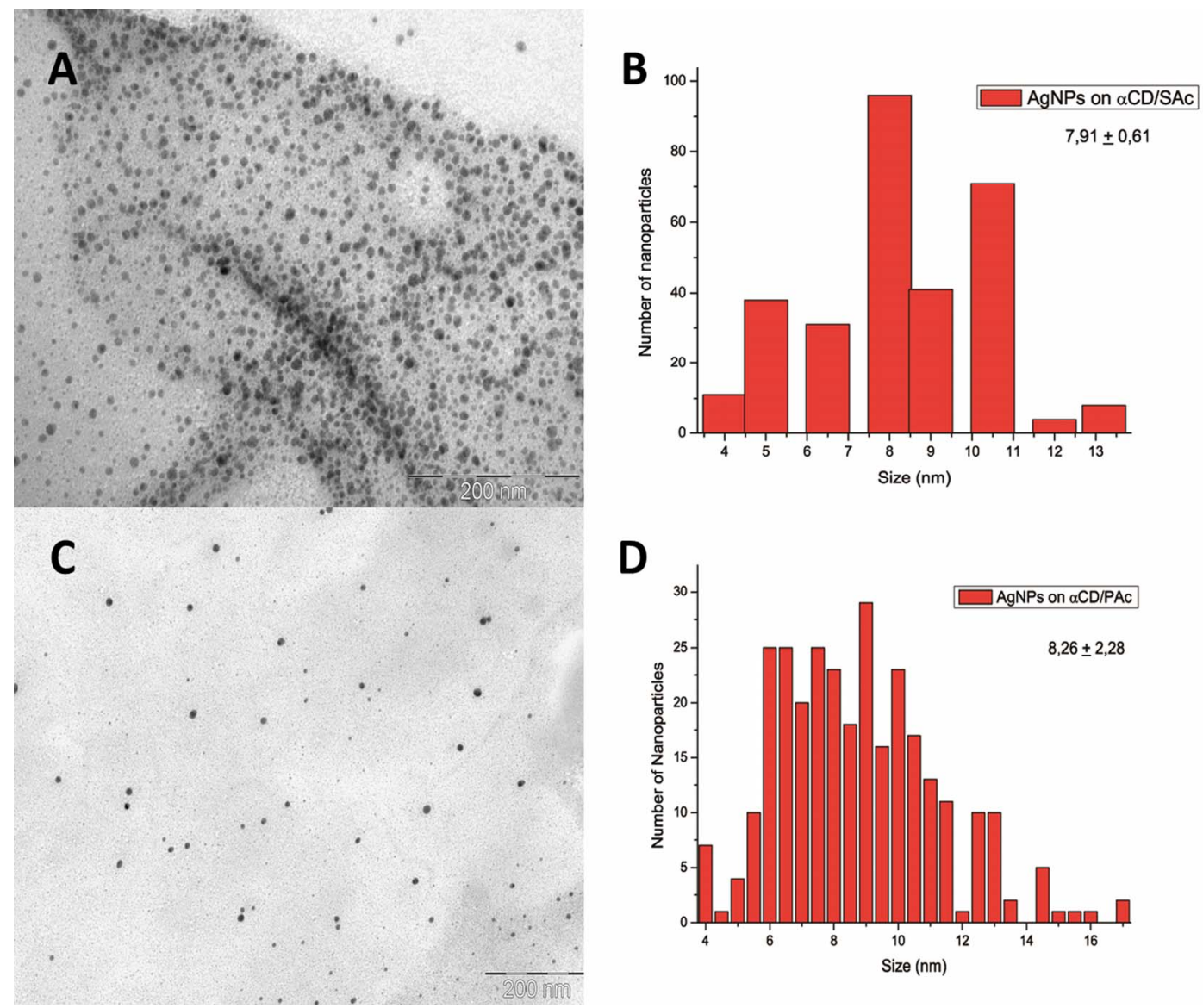

Figure 4. (A) and (B) corresponds to TEM image of AgNPs obtained onto $\alpha$-CD/SAc IC and its particle size distribution histogram, respectively. (C) TEM image of AgNPs obtained onto crystal of $\alpha$-CD/PAc IC and (D) correspond to respective histogram.

gold and $-\mathrm{COOH}$ groups because gold deposition experiments using the sputtering technique onto the $\alpha-\mathrm{CD}$ IC with carboxylic acids guests resulted in the deposition of macrocrystalline gold onto the surface of the IC crystals. This result indicates that nanoparticles were absent, which was evidenced by the absence of the characteristic plasmon resonance for gold nanoparticles.

\subsection{TEM Analysis of AgNPs}

TEM images of AgNPs obtained by the sputtering technique are shown in Figure 4. Histograms with distribution functions for the analysis of the size of AgNPs resulted in the determination of the particle size being 7.91 $\pm 0.61 \mathrm{~nm}$ when $\alpha-\mathrm{CD} / \mathrm{SAc}$ was used and $8.26 \pm 2.28 \mathrm{~nm}$ for $\alpha-\mathrm{CD} / \mathrm{PAc}$.

The AgNPs show low dispersion in size. In this case the average size of these particles was approximately 8 $\mathrm{nm}$ when were used $\alpha-\mathrm{CD} / \mathrm{SAc}$ and $\alpha-\mathrm{CD} / \mathrm{PAc}$ IC. In both cases, the same conditions of pressure, current and deposition time were used, and therefore it does not follow a relationship between the carboxylic acid used as the guest molecule and the nanoparticle sizes obtained, considering that there is a minimum difference between the lengths of the alkyl chains of the two carboxylic acids used.

\section{Conclusions}

The self-assembly of AgNPs onto the preferred face of $\alpha-\mathrm{CD} /$ carboxylic acid IC microcrystals by the magnetron sputtering technique was used as a new method for for nucleation and epitaxial growth of these nanoparticles on the surface of compounds including carboxylic acids containing as guest molecules.

Preferential decoration of the $\{001\}$ plane of the $\alpha-\mathrm{CD}$ IC crystal occurred because the $-\mathrm{COOH}$ groups of the guest molecules within the $\alpha$-CD protruded into this plane. These $-\mathrm{COOH}$ groups formed a two-dimensional hexagonal lattice that interacted with the metal nanoparticles, which stabilized and arranged the particles in an orderly manner. The magnetron sputtering technique used to prepare the ordered AgNPs has several benefits such as the lack of a waste stream and scalability, which are attractive advantages for industrial applications, no use of solvents and environmentally-friendly. This work 
provides evidence that assemblies of silver nanoparticles selectively formed by magnetron sputtering were mediated by a guest molecule in the crystals of a supra-molecular structure of cyclodextrin inclusion compounds.

\section{Acknowledgements}

This work is supported by Projects Fondecyt 1080505, 1090029, 1090143. B.H.I. acknowledges CONICYT for a PhD fellowship and Dr. Fernando Albericio for their support and funding and Serveis Cientificotècnics Universitat de Barcelona (SCT-UB) for electron microscopy analysis.

\section{REFERENCES}

[1] B. Herrera, C. Adura, P. Jara, N. Yutronic and M. J. Kogan, "Selective Nanodecoration of Modified Cyclodextrin Crystals with Gold Nanorods," Journal of Colloid and Interface Sciences, Vol. 389, No. 1, 2013, pp. 42-45. doi:10.1016/j.jcis.2012.08.027

[2] M. Rycenga, P. H. C. Camargo, W. Li, C. H. Moran and Y. Xia "Understanding the SERS Effects of Single Silver Nanoparticles and Their Dimers, One at a Time," Journal of Physical Chemistry Letters, Vol. 1, No. 4, 2010, pp. 696-703. doi:10.1021/jz900286a

[3] S. D. Standridge, G. C. Schatz and J. T. Hupp, "Toward Plasmonic Solar Cells: Protection of Silver Nanoparticles via Atomic Layer Deposition of $\mathrm{TiO}_{2}$," Langmuir, Vol. 25, No. 5, 2009, pp. 2596-2600. doi:10.1021/la900113e

[4] T. Ghodselahi, T. N. Nejad, M. A. Vesaghi, K. Z. Salimi and H. Mobasheri, "Synthesis of Silver Nanoparticles Array and Application of Their Localized Surface Plasmon Resonance in Biosensor Design," International Conference on Nanotechnology and Biosensors IPCBEE, Vol. 2, Singapore, 2011, pp. 59-61.

[5] S. Ravindra, Y. M. Mohan, N. N. Reddy and K. M. Raju, "Fabrication of Antibacterial Cotton fibres Loaded with Silver Nanoparticles via Green Approach," Colloids and Surfaces A: Physicochemical and Engineering Aspects, Vol. 367, No. 1-3, 2010, pp. 31-40. doi:10.1016/j.colsurfa.2010.06.013

[6] Y. Fujiki, N. Tokunaga and S. S. Sada, "Anisotropic Decoration of Gold Nanoparticles onto Specific Crystal Faces of Organic Single Crystals," Angewandte Chemie International Edition, Vol. 45, No. 29, 2006, pp. 4764 -4767. doi:10.1002/anie.200601470

[7] Y. Fujiki, S. Shinkay and K. Sada, "Selective Deposition of Metal Complex Nanocrystals onto the Surfaces of Organic Single Crystals Bearing Pyridine Moieties," Crystal Growth and Design, Vol. 9, No. 6, 2009, pp. 2751-2755. doi: $10.1021 / \operatorname{cg} 801384 \mathrm{c}$

[8] K. A. Connors, "The Stability of Cyclodextrin Complexes in Solution”, Chemical Reviews, Vol. 97, No. 5, 1997, pp.
1325-1357. doi:10.1021/cr960371r

[9] T. Loftsson and D. Duchêne, "Cyclodextrins and Their Pharmaceutical Applications," International Journal of Pharmaceutics, Vol. 329, No. 9, 2007, pp. 1-11. doi:10.1016/j.ijpharm.2006.10.044

[10] M. R. Caira, "On the Structurality of Cyclodextrin Inclusion Complexes and Its Practical Utility," Revue Roumaine de Chimie, Vol. 46, No. 4, 2001, pp.371-386.

[11] Z. Dang, L. X. Song, X.Q. Guo, F.Y Du, J. Yang and J. Yang, "Aplications of Powder X-Ray Diffraction to Inclusion Complexes of Cyclodextrins," Current Organic Chemistry, Vol. 15, No. 6, 2011, pp. 848-861. http://dx.doi.org/10.2174/138527211794518899

[12] J, Szejpli, "Introduction and General Overview of Cyclodextrin Chemistry," Pure and Applied Chemistry, Vol. 98, No. 5, 1998, pp. 1743-1754.

[13] P. Jara, L. Barrientos, B. Herrera and I. Sobrados, "Inclusion Compounds of $\alpha$-Cyclodextrin with Alkylthiols," Journal of the Chilean Chemical Society, Vol. 53, No. 2, 2008, pp. 1399-1401. doi:10.4067/S0717-97072008000200005

[14] P. Jara, X. Cañete, V. Lavayén and N. Yutronic, "Inclusion compounds of $\alpha$ - and $\gamma$-Cordially,yclodextrins with n-Alkyl Amine (n = 12,18)," Journal of the Chilean Chemical Society, Vol. 49, No. 3, 2004, pp. 241-244. doi: 10.4067/S0717-97072004000300010

[15] S. Rodríguez-Llamazares, N. Yutronic, P. Jara, U. Englert, M. Noyong and U. Simon, "The Structure of the First Supramolecular $\alpha$-Cyclodextrin Complex with an Aliphatic Monofunctional Carboxylic Acid," European Journal of Organic Chemistry, Vol. 2007, No. 26, 2007, pp. 42984300. doi:10.1002/ejoc.200700454

[16] L. Barrientos, N. Yutronic, F. del Monte, M. C. Gutiérrez and P. Jara, "Ordered Arrangement of GoId Nanoparticles on $\alpha$-Cyclodextrins-Dodecanethiol Inclusion Compound Produced by Magnetron Sputering," New Journal of Chemistry, Vol. 31, No. 8, 2007, pp. 1400-1402. http://dx.doi.org/10.1039/b706346f

[17] L. Barrientos, P. Allende, C. Orellana and P. Jara, “Ordered Arrangements of Metal Nanoparticles on Alphacyclodextrin Inclusion Complexes by Magnetron Sputtering," Inorganica Chimica Acta, Vol. 380, No. 1, 2012, pp. 372-377. doi:10.1016/j.ica.2011.10.032

[18] S. Rodríguez-Llamazares, P. Jara, N. Yutronic, M. Noyong, M. Fischler and U. Simon, "Preferential Adhesion of Silver Nanoparticles onto Crystal Faces of $\alpha$ Cyclodextrin/Carboxylic Acids Inclusion Compounds," Journal of Nanoscience and Nanotechnology, Vol. 12, No. 12, 2012, pp. 8929-8934.

[19] Y. Tan, Y. Wang, L. Jiang and D. Zhu, "Thiosalicylic Acid-Functionalized Silver Nanoparticles Synthesized in One-Phase System," Journal of Colloid and Interface Science, Vol. 249, No. 2, 2002, pp. 336-345. doi:10.1006/jis. 2001.8166 


\section{Supplementary Material}

\section{Nuclear Magnetic Resonance $\left({ }^{1} \mathrm{HNMR}\right)$ of $\alpha$-CD/carboxylic acids Inclusion Compounds}

Experiments of proton Nuclear Magnetic Resonance ( ${ }^{1} \mathrm{HNMR}$ ) Spectroscopy were realized in FT/400 MHz NMR Spectrometer, using TMS like internal reference. The DMSO-d6 solvent was used.

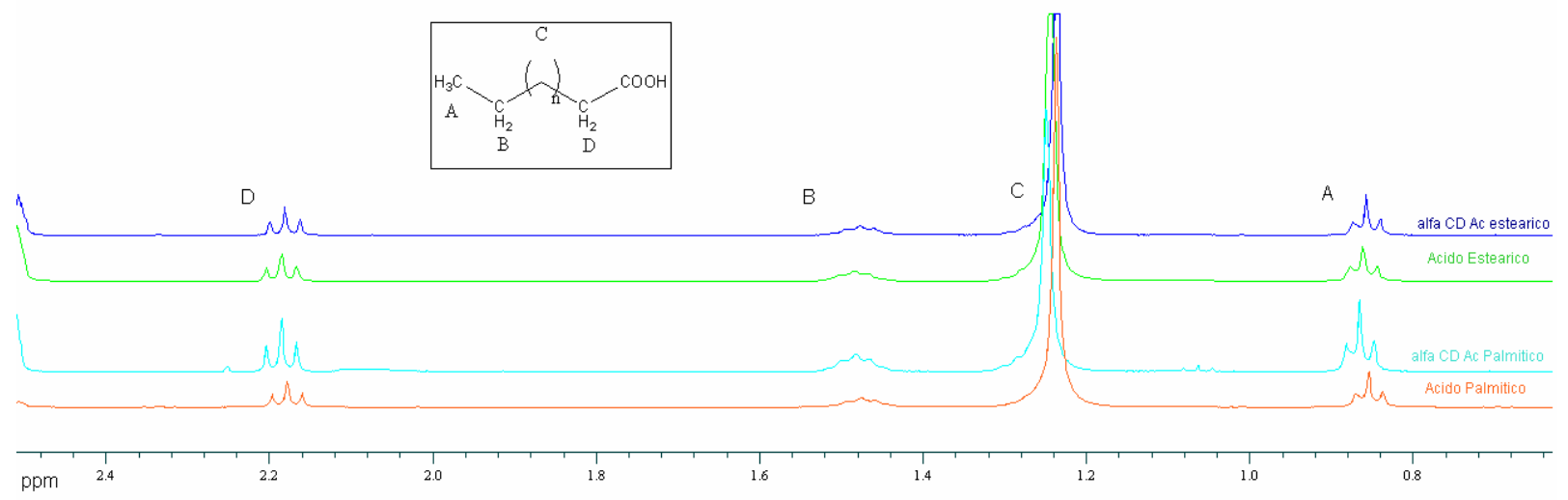

Figure S1. ${ }^{1}$ HNMR spectrums of guest acid inside the inclusion compound compared with pure carboxyl acid molecules.

Table S1. ${ }^{1}$ HNMR chemical shifts $(\delta)$, of protons in pure guests and cyclodextrin interacting with different guests and their complexation shifts $\left(\Delta \delta=\delta_{\text {pure guest }}-\delta_{\text {included guest }}\right)$.

\begin{tabular}{|c|c|c|c|c|c|c|c|}
\hline $\mathrm{H}$ & $\delta$ PAc. (ppm) & $\begin{array}{c}\delta \alpha-\mathrm{CD} / \mathrm{Pac} \\
(\mathrm{ppm})\end{array}$ & $\Delta \delta(\mathrm{ppm})$ & $\mathrm{H}$ & $\delta \mathrm{SAc}(\mathrm{ppm})$ & $\begin{array}{c}\delta \alpha-\mathrm{CD} / \mathrm{SAc} \\
(\mathrm{ppm})\end{array}$ & $\Delta \delta(\mathrm{ppm})$ \\
\hline $\mathrm{CH} 3$ & 0.854 & 0.858 & 0.004 & $\mathrm{CH} 3$ & 0.853 & 0.852 & 0.001 \\
\hline $\mathrm{CH} 2$ & 1.476 & 1.477 & 0.001 & $\mathrm{CH} 2$ & 1.475 & 1.471 & 0.004 \\
\hline$(\mathrm{CH} 2) \mathrm{n}$ & 1.237 & 1.239 & 0.002 & $(\mathrm{CH} 2) \mathrm{n}$ & 1.235 & 1.236 & 0.001 \\
\hline $\mathrm{CH} 2$ & 2.177 & 2.182 & 0.005 & $\mathrm{CH} 2$ & 2.176 & 2.174 & 0.002 \\
\hline $\mathrm{COOH}$ & 11.976 & 11.975 & 0.001 & $\mathrm{COOH}$ & 11.931 & 11.97 & 0.039 \\
\hline
\end{tabular}

Table S2. ${ }^{1}$ HNMR chemical shifts of most important protons in pure $\alpha$-cyclodextrin and $\alpha$-cyclodextrin protons interacting with different guests and their complexation shifts $\left(\Delta \delta=\delta_{\text {pure-CD }} \delta_{\text {complex }}\right)$.

\begin{tabular}{|c|c|c|c|c|c|c|c|}
\hline $\mathrm{H}$ & $\alpha-\mathrm{CD}(\mathrm{ppm})$ & $\alpha-\mathrm{CD} / \mathrm{PAc}(\mathrm{ppm})$ & $\Delta \delta(\mathrm{ppm})$ & $\mathrm{H}$ & $\alpha-\mathrm{CD}(\mathrm{ppm})$ & $\alpha-\mathrm{CD} / \mathrm{Sac}(\mathrm{ppm})$ & $\Delta \delta(\mathrm{ppm})$ \\
\hline H3 & 3.77 & 3.775 & 0.005 & $\mathrm{H} 3$ & 3.77 & 3.774 & 0.004 \\
\hline H5 & 3.582 & 3.59 & 0.008 & H5 & 3.582 & 3.612 & 0.03 \\
\hline H6 & 3.634 & 3.646 & 0.012 & $\mathrm{H} 6$ & 3.634 & 3.051 & 0.583 \\
\hline
\end{tabular}

\section{Energy Dispersive X-ray Spectroscopy (EDAX)}

EDAX analyses were acquired with a FEI SEM with a Quantum Detector 200 EDAX GENESIS Model. 


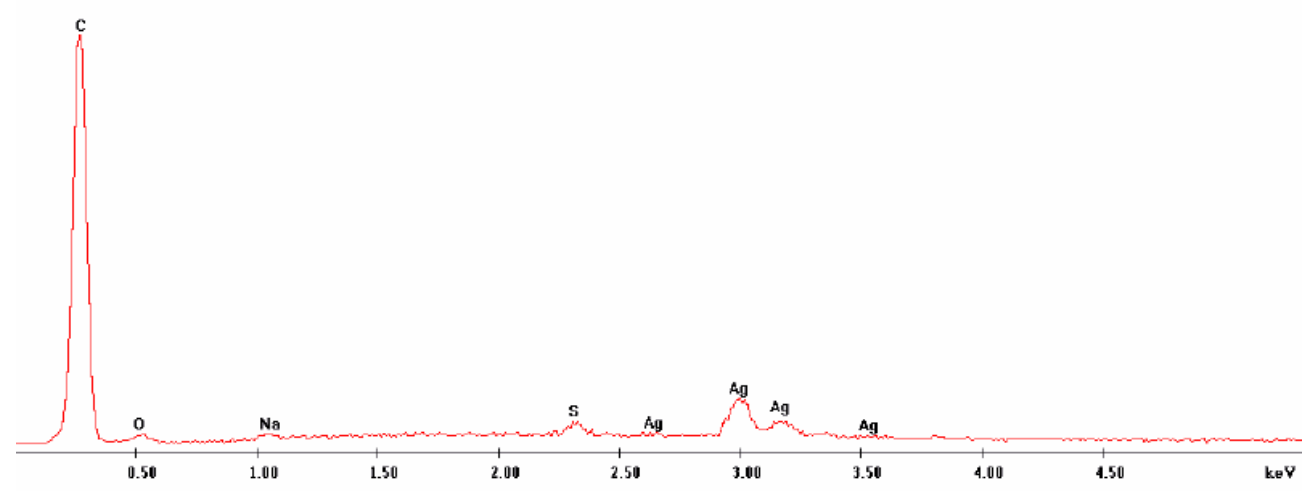

Figure S2. EDAX analysis of $\alpha$-CD/SAc IC surface nanodecorated with AgNPs.

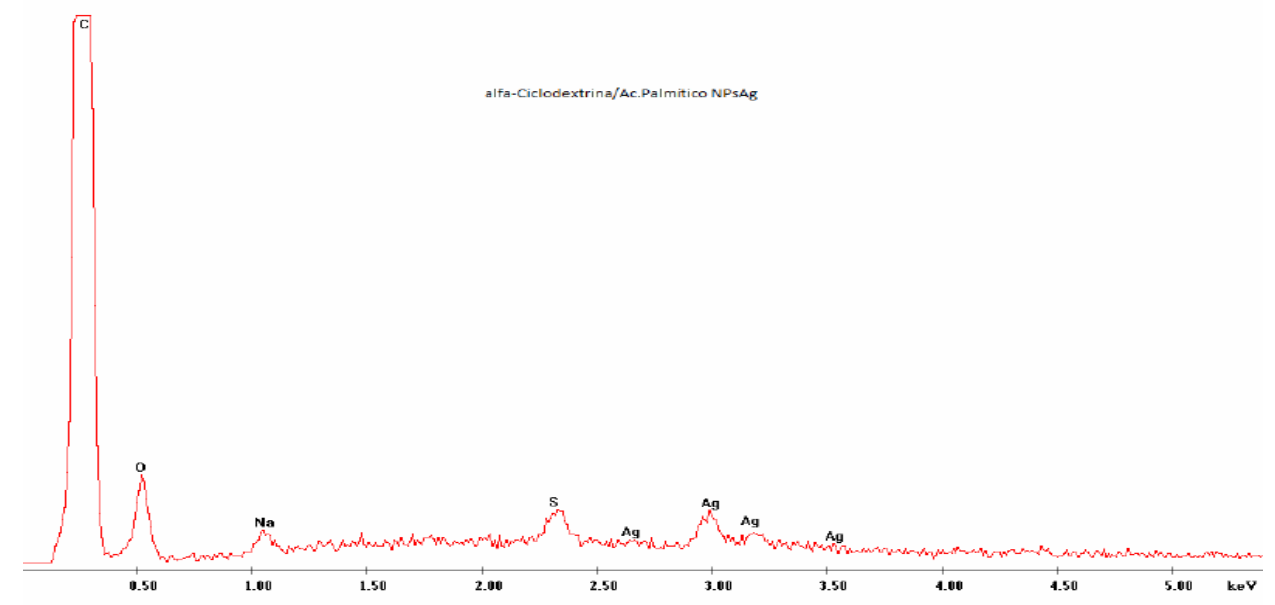

Figure S3. EDAX analysis of $\alpha$-CD/PAc IC surface nanodecorated with AgNPs.

The analysis of carbon ribbon was performed was performed to account for the presence of $\mathrm{Na}$ and $\mathrm{S}$ elements.

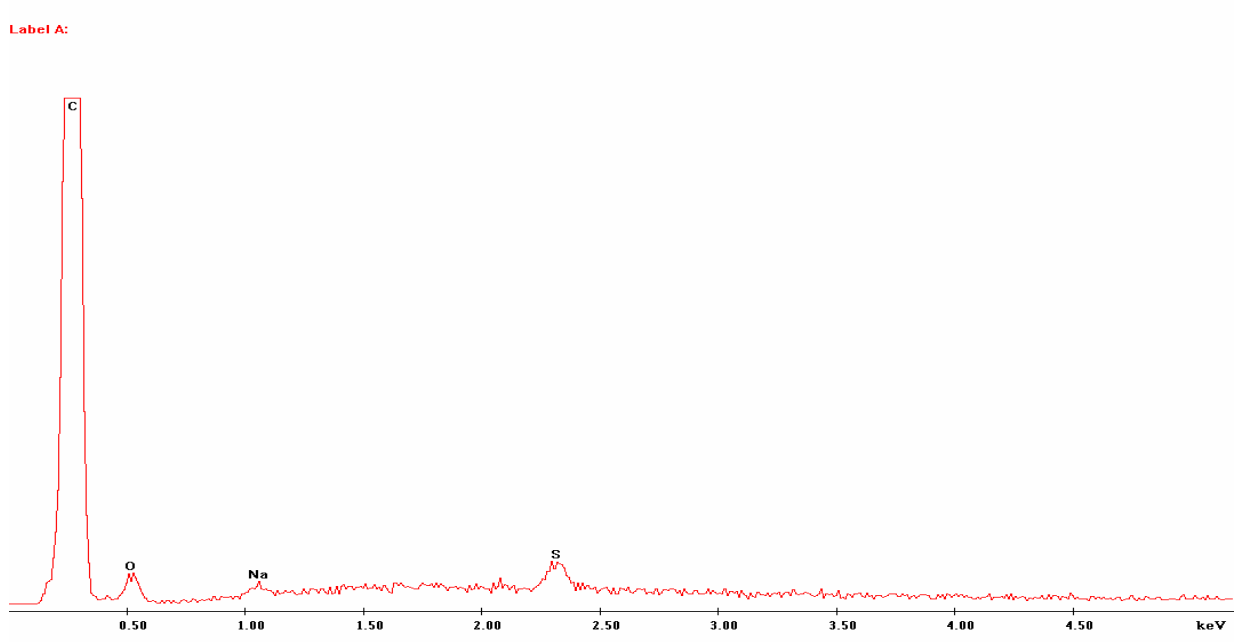

Figure S4. EDAX analysis performed onto the carbon ribbon. 


\section{Powder X-Ray Diffraction}

Diffractometer Siemens D-5000 Equipment with radiation $\mathrm{Cu}-\mathrm{Ka}$ (49 kV and $30 \mathrm{~mA})$, provided with a graphite monocromador $\left(\lambda=1.5418 \AA\right.$ ) was used .Range of sweep between $5^{\circ} \geq \theta \geq 80^{\circ}$.
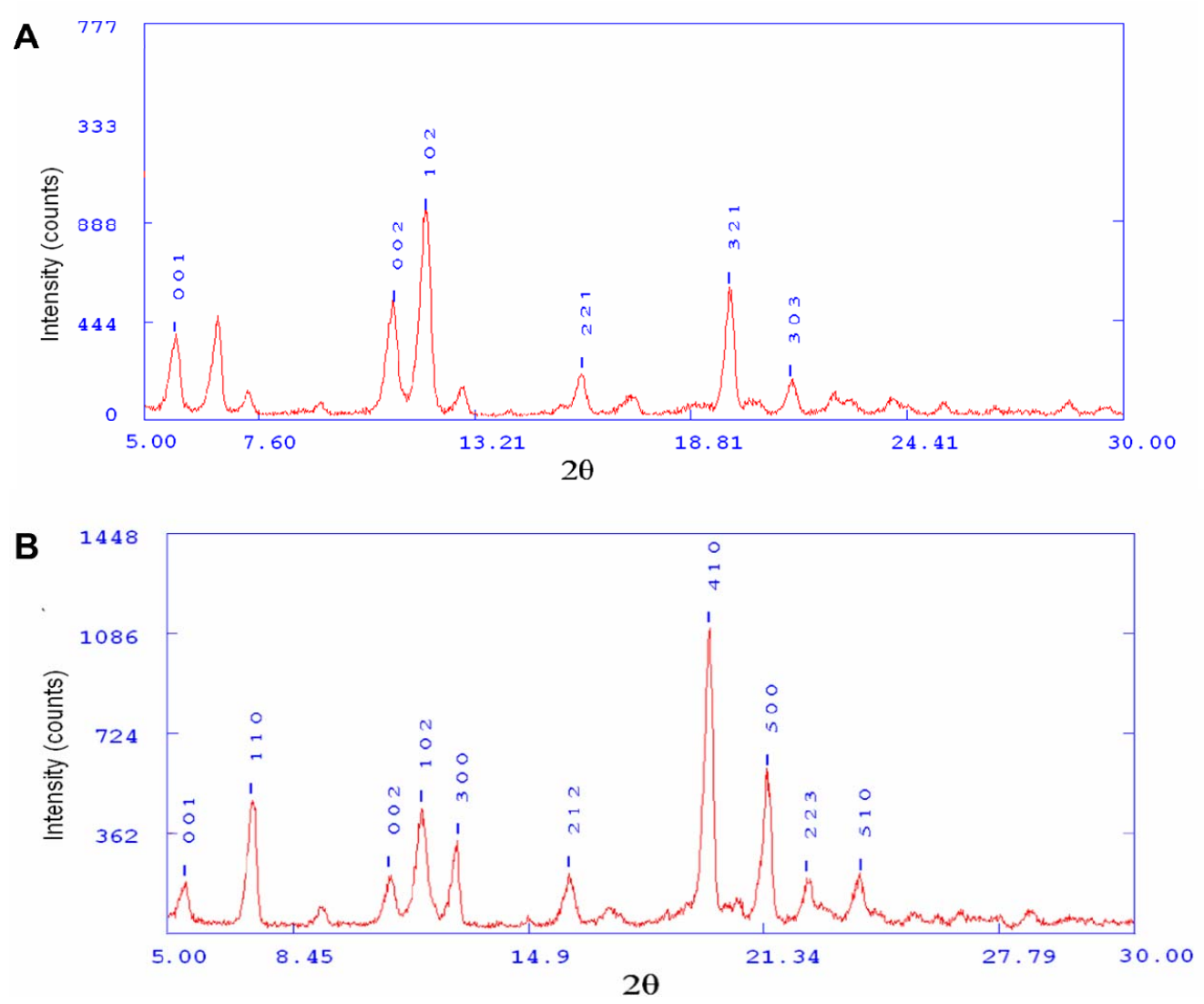

Figure S5. A. Diffractogram of $\alpha-\mathrm{CD} / \alpha-\mathrm{CD} / \mathrm{SAc}$ and B. Diffractogram of $\alpha-\mathrm{CD} / \alpha-\mathrm{CD} / \mathrm{PAc}$.

All peaks in the diffractogram were indexed on the basis of a hexagonal lattice with parameters to $\alpha=\beta=90^{\circ}, \gamma=120^{\circ}$, $\mathrm{a}=\mathrm{b}=23.700 \AA$ and $\mathrm{c}=15.900 \AA$. 